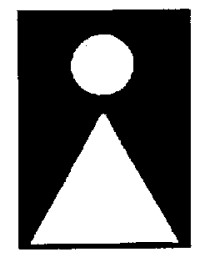

NATIONAL

OPTICAL

ASTRONOMY

OBSERVATORY

Preprint Series

NOAO Preprint No. 884

The Age Of The Inner Halo Globular Cluster NGC 6652

\author{
Brian Chaboyer \\ (Department of Physics and Astronomy, Dartmouth College) \\ Ata Sarajedini \\ (Astronomy Department, Wesleyan University, Middletown, CT) \\ and \\ Taft E. Armandroff \\ (National Optical Astronomy Observatory)
}

To appear in: The Astronomical Journal, December 2000 Issue 


\title{
The Age of the Inner Halo Globular Cluster NGC $6652^{1}$
}

\author{
Brian Chaboyer \\ Department of Physics and Astronomy, Dartmouth College, 6127 Wilder Laboratory, Hanover, \\ NH, USA 03755-3528 \\ Brian.Chaboyer@Dartmouth.edu \\ Ata Sarajedini ${ }^{2}$ \\ Astronomy Department, Wesleyan University, Middletown, CT 06459 \\ ata@astro.wesleyan.edu \\ and \\ Taft E. Armandroff \\ National Optical Astronomy Observatory, P.O. Box 26732, Tucson, AZ 85726 \\ armandenoao.edu
}

\begin{abstract}
HST $(V, I)$ photometry has been obtained for the inner halo globular cluster NGC 6652. The photometry reaches $\sim 4$ mag below the turn-off and includes a well populated horizontal branch (HB). This cluster is located close to the Galactic center at $\mathrm{R}_{\mathrm{GC}} \simeq$ $2.0 \mathrm{kpc}$ with a reddening of $\mathrm{E}(V-I)=0.15 \pm 0.02$ and has a metallicity of $[\mathrm{Fe} / \mathrm{H}] \simeq$ -0.85 . Based upon $\Delta \mathrm{V}_{\mathrm{HB}}^{\mathrm{SGB}}, \mathrm{NGC} 6652$ is $11.7 \pm 1.6 \mathrm{Gyr}$ old. Using $\Delta \mathrm{V}_{\mathrm{HB}}^{\mathrm{SGB}}$ precise differential ages for 47 Tuc (a thick disk globular), M107 and NGC 1851 (both halo clusters) were obtained. NGC 6652 appears to be the same age as 47 Tuc and NGC 1851 (within $\pm 1.2 \mathrm{Gyr}$ ), while there is a slight suggestion that M107 is older than NGC 6652 by $2.3 \pm 1.5 \mathrm{Gyr}$. As this is a less than $2 \sigma$ result, this issue needs to be investigated further before a definitive statement regarding the relative age of M107 and NGC 6652 may be made.
\end{abstract}

Subject headings: color-magnitude diagrams - Galaxy: formation - globular clusters: general - globular clusters: individual (NGC 6652)

\footnotetext{
${ }^{1}$ Based on observations made with the NASA/ESA Hubble Space Telescope obtained at the Space Telescope Science Institute, which is operated by the Association of Universities for Research in Astronomy, Incorporated, under NASA contract NAS5-26555.

${ }^{2}$ Guest User, Canadian Astronomy Data Centre, which is operated by the Dominion Astrophysical Observatory for the National Research Council of Canada's Herzberg Institute of Astrophysics.
} 


\section{Introduction}

NGC 6652 is a compact globular cluster which is projected near the Galactic center at $\ell=1.53^{\circ}$ and $b=-11.38^{\circ}$ (Harris 1996). A color magnitude diagram (CMD) for this cluster was presented by Ortolani, Bica \& Barbuy (1994, hereafter OBB), who determined a number of cluster parameters, including $[\mathrm{Fe} / \mathrm{H}] \approx-0.9, \mathrm{E}(B-V)=0.10 \pm 0.02,\left\langle\mathrm{~V}_{\mathrm{HB}}>=15.85 \pm 0.04\right.$ and $\Delta \mathrm{V}_{\mathrm{HB}}^{\mathrm{TO}}=3.35 \pm 0.16$. The small value of $\Delta \mathrm{V}_{\mathrm{HB}}^{\mathrm{TO}}$ suggested that NGC 6652 is younger than the average Galactic halo cluster. Adopting an absolute horizontal branch (HB) magnitude of $\mathrm{M}_{\mathrm{V}}^{\mathrm{HB}}=+0.7$, OBB determined $\mathrm{R}_{\mathrm{GC}}=2.1 \mathrm{kpc}$. Its proximity to the Galactic center, reasonably low metallicity and small reddening make NGC 6652 somewhat unique. It is an inner halo globular cluster for which one can obtain an accurate relative age with respect to other halo clusters.

Based upon its metallicity, radial velocity, HB type and position in the Galaxy, Zinn (1993) classified NGC 6652 as an old halo cluster. This is at odds with OBB's suggestion (based on the turn-off magnitude) that NGC 6652 is younger than the average halo cluster. Because NGC 6652 is a compact cluster located in a crowded field, the photometry near the turn-off obtained by OBB had a great deal of scatter leading to a large error in the age determination. For this reason, we were granted HST time to obtain a deep CMD of NGC 6652, in order to clearly delineate the turn-off region and to obtain an accurate estimate of its age. The observations and data reduction procedure are presented in $\S 2$ and the generation of the isochrones used to determine the age of NGC 6652 is discussed in $\S 3$. The analysis of the CMD is presented in $\S 4$, the discussion of the age of NGC 6652 is in $\S 5$, and the summary of the results are in $\S 6$.

\section{Observations and Data Reduction}

NGC 6652 was observed in September 1997 with WFPC2 on HST. The cluster was centered on the PC1 chip. Observations were obtained with the F555W (V) filter $(3 \times 23 \mathrm{~s}$ and $12 \times 160 \mathrm{~s})$ and the F814W (I) filter $(3 \times 20 \mathrm{~s}$ and $12 \times 160 \mathrm{~s})$. The short exposure times were chosen to ensure good photometry at the level of the $\mathrm{HB}$, while the long exposure times were picked to ensure good photometry around the turn-off and to allow for a good inter-comparison between the long and short exposure frames. Figure 1 shows the averaged, long exposure F555W image of the cluster. The WFPC2 images of NGC 6652 were divided into three sets of four long exposure (160s) F555W and F814W frames and one set of three short exposure F555W (23s) and F814W (20s) frames. Each set of four long and three short exposures in a given filter was averaged using GCOMBINE in IRAF/STSDAS with cosmic ray rejection enabled and the data quality files used to flag defective pixels. This yielded a total of 8 WFPC2 images which were input into our photometric procedure.

We utilized the aperture photometry routines in DAOPHOT II (Stetson 1987, 1994) adopting an aperture radius of 2.5 pixels in the Planetary Camera and 2 pixels in the Wide Field chips. Corrections from the small aperture photometry to the standard 0.5 arcsec radius were determined using 30 to 50 relatively uncrowded stars. These gave aperture corrections with typical standard 
deviations between 0.01 and $0.03 \mathrm{mag}$. The $\mathrm{V}$ and I instrumental magnitudes were matched to form colors, and corrected to a 0.5 arcsec radius aperture. The total instrumental magnitudes were then adjusted for exposure time and placed on the standard VI system using the coefficients in Table 7 of Holtzman et al. (1995). A correction was also applied for the well-known charge transfer efficiency (CTE) problem using the formulation of Whitmore, Heyer \& Casertano (1999). Magnitudes of stars in common between the long exposure frames were averaged together and only stars measured on all three frame pairs were retained. The final photometry file contains stars fainter than $V=17$ from the long exposure frames and brighter than $V=17$ from the short exposures (4832 stars in total). In order to ensure that the long and short exposures magnitudes were internally consistent, the magnitude of a number of stars (approximately 10 per chip) in common between the long and short exposures were compared and the average offset determined. This average offset $(0.03 \mathrm{mag})$ was then applied to the short exposure magnitudes, implying that all of the magnitudes are on the long exposure scale. The photometry data is presented in Table 1, the full version of which is available in the electronic version of this article.

Due to its compact nature, the inner regions of NGC 6652 are crowded on the PC1 chip. The faint (long exposure) photometry within $r<11.5^{\prime \prime}$ (250 pixels) of the cluster center showed considerable scatter around the turn-off region. For this reason, the long exposure photometry in our final CMD only includes stars with $r>11.5^{\prime \prime}$ from the cluster center. This trimmed data-set includes 3790 stars with good photometry.

A careful inspection of the photometry obtained with the different chips showed no difference $[\delta(V-I)<0.005]$ in the location of the principal features (i.e. location of the main sequence, sub-giant branch, red giant branch and the HB) in the CMD. This indicates that the aperture corrections (applied to each chip individually) are accurate, implying that the internal errors in the photometry are very small. The external errors are set by the accuracy of the Holtzman et al. (1995) transformations to the standard system.

\section{The Stellar Models and Isochrones}

Stellar evolution tracks for masses in the range $0.50-1.1 M_{\odot}$ (in $0.05 M_{\odot}$ increments) were calculated using the Yale stellar evolution code (Guenther et al. 1992). These models incorporate the following physics: high temperature opacities from Iglesias \& Rogers (1996); low temperature opacities from Kurucz (1991); nuclear reaction rates from Bahcall \& Pinsonneault (1992) and Bahcall (1989); helium diffusion coefficients from Michaud \& Proffitt (1993); and an equation of state which includes the Debye-Hückel correction (Guenther et al. 1992). Note that this equation of state yields stellar models which are in good agreement with those derived using the OPAL equation of state (Rogers 1994; Chaboyer \& Kim 1995). The surface boundary conditions were calculated using a grey $T-\tau$ relation. The stellar models employ a solar calibrated mixing length $\left(\alpha_{\odot}=1.78\right)$. The models were typically evolved from the zero age main sequence to the upper giant branch (around $M_{V} \approx-1.0$ ) in 4000 time steps. In each time step, the stellar evolution 
equations were solved with a numerical accuracy exceeding $0.01 \%$. The models did not include any overshooting beyond the formal edge of the convection zones.

The heavy element composition of the models was chosen to reflect the observed abundance ratios in metal-poor stars (e.g., Lambert 1989). In particular, the $\alpha$-capture elements $(\mathrm{O}, \mathrm{Mg}, \mathrm{Si}$, $\mathrm{S}$, and $\mathrm{Ca}$ ) and $\mathrm{Ti}$ were enhanced by $+0.4 \mathrm{dex}$, while $\mathrm{Mn}$ was made to be deficient by the same proportions. The solar abundances (before $\alpha$-enhancement) were taken from Grevesse \& Noels (1993). Kurucz (low temperature) and Iglesias \& Rogers (high temperature) provided us with opacities for this specific mixture.

NGC 6652 has $[\mathrm{Fe} / \mathrm{H}]=-0.96$ on the Zinn \& West (1984) scale and $[\mathrm{Fe} / \mathrm{H}]=-0.85$ on the Carretta \& Gratton (1997) scale. Based upon the inclination of the red giant branch, OBB found that NGC 6652 was more metal-poor than $47 \mathrm{Tuc}$, and concluded that $[\mathrm{Fe} / \mathrm{H}] \approx-0.9$. In light of these abundance determinations, stellar models and isochrones were calculated for $[\mathrm{Fe} / \mathrm{H}]=$ $-1.20,-1.00,-0.85,-0.70$. The most metal-poor isochrone was calculated to facilitate an age comparison with somewhat more metal-poor halo clusters (see §5.2). To explore the effect of the $\alpha$-element enhancement, at $[\mathrm{Fe} / \mathrm{H}]=-0.85$, scaled solar abundance models and isochrones were calculated in addition to the $\alpha$-element enhanced isochrones.

Our calibrated solar model had an initial solar helium abundance of $Y_{\odot}=0.263$ and heavy element mass fraction of $Z_{\odot}=0.0182$. Assuming a primordial helium abundance of $Y_{p}=0.234$ (Olive et al. 1997), our calibrated solar model implies $\Delta Y / \Delta Z=1.59$. Thus, the helium abundance for the models was determined using

$$
Y=0.234+1.59 \cdot Z
$$

There is some evidence that 47 Tuc has a solar helium abundance (Salaris \& Weiss 1998). As 47 Tuc is being used as a comparison cluster in this study, for $[\mathrm{Fe} / \mathrm{H}]=-0.70$ stellar models and isochrones were calculated for a solar helium abundance $\left(Y_{\odot}=0.263\right)$ in addition to the helium abundance implied by equation (1), $Y=0.245$. The evidence for a solar helium abundance in 47 Tuc is not strong, and indeed Dorman, Vandenberg \& Laskarides (1989) found $Y \approx 0.24$ from fitting the HB morphology.

The isochrones were constructed by interpolating among the evolutionary tracks using the method of equal evolutionary points (Prather 1976) for the age range 6 - 18 Gyr, in 1 Gyr increments. The isochrones were transformed from the theoretical $\left(\log L, \log \mathrm{T}_{\text {eff }}\right)$ plane to observed colors and magnitudes using color transformations and bolometric corrections based on the Kurucz (1993) model atmospheres. The transformations used here were kindly supplied to us by Sukyoung Yi (private communication), who derived them for a wide range of metallicities, using a procedure similar to that described by Bessell et al. (1998). At solar metallicities, the Yi tables agree with Bessell et al. (1998) Table 1 to within $\sim 0.007 \mathrm{mag}$ in $B-V$ and $\sim 0.004$ in $V-I$ over the range of colors appropriate for old clusters. In the Yi tables, the solar colors are $(B-V)_{\odot}=0.670$ and $(V-I)_{\odot}=0.718$. Bessell et al. (1998) extensively tested their Kurucz-based (ATLAS9) colors for solar metallicities, and found that all indices agreed extremely well with observations for 
$\log \mathrm{T}_{\text {eff }}>4250 \mathrm{~K}(4250 \mathrm{~K}$ corresponds to $V-I \simeq 1.3$ at $[\mathrm{Fe} / \mathrm{H}]=-0.85)$.

Weiss \& Salaris (1999) recently studied the issue of color transformations for isochrones in the VI-plane. They concluded that on the main sequence the color transformations based on the ATLAS9 models were a good choice. On the giant branch Weiss \& Salaris (1999) preferred to use empirical color relations. However, an inspection of their Figure 5 indicates that the color transformation based upon the ATLAS9 models is in good agreement with the empirical relations preferred by Weiss \& Salaris (1999). Thus it appears that our choice of the Yi color table is a reasonable one, in good agreement with presently available data.

Figure 2 displays the 12 Gyr isochrones for the various compositions used in this paper. It is interesting to note that changing the helium abundance from $Y=0.245$ (given by eqn. (1)) to the solar value $\left(Y_{\odot}=0.263\right)$, has very little effect on the isochrone. Thus, the exact choice of $Y$ will not effect the derived ages. In contrast, changing the $[\alpha / \mathrm{Fe}]$ ratio by 0.4 dex has a substantial effect on the isochrones (larger than changing $[\mathrm{Fe} / \mathrm{H}]$ by $0.15 \mathrm{dex}$ ) and so the uncertainty in the actual value of $[\alpha / \mathrm{Fe}]$ in NGC 6652 will lead to a substantial uncertainty in the derived age.

In $§ 5.1$, the isochrones are fit to the CMD of NGC 6652. This fitting procedure assumes that the location of the unevolved main sequence predicted by the theoretical isochrones is accurate. To test this assumption, the Hipparcos catalogue was searched for stars which (a) have $\sigma_{\pi} / \pi<$ 0.10 , (b) are fainter than $\mathrm{M}_{\mathrm{V}} \simeq 5.5$, and (c) are not known or suspected Hipparcos binaries or variables. This resulted in a list of 2618 stars, of which the great majority have near solar metallicity. As we are interested in stars with a similar metallicity to NGC 6652, we require stars with $-1.05 \leq[\mathrm{Fe} / \mathrm{H}] \lesssim-0.65$. To identify these metal-poor stars in the Hipparcos sample, we have cross identified the above Hipparcos subsample with the 1996 high resolution spectroscopic catalogue of Cayrel de Strobel et al. (1997), and more recent papers which give high dispersion abundance analysis of metal-poor stars: Reid (1998); Clementini et al. (1999); Tomkin \& Lambert (1999); Carretta, Gratton \& Sneden (2000). Any stars which were known or suspected binaries and whose companions would contribute a significant flux in the BVI passbands were removed from our final list. In total only 3 stars in the Hipparcos catalogue pass our stringent selection criteria. A comparison between these stars and our isochrones is shown in Figure 3. Unfortunately, we were unable to locate Kron-Cousins $V-I$ colors for these stars, so the comparison is only shown in $B-V$. The small number of stars prevents us from reaching any definitive conclusions based upon this comparison. However, we note that the star with the most accurate parallax (with $[\mathrm{Fe} / \mathrm{H}]=-0.87$ ) lies close to our $[\mathrm{Fe} / \mathrm{H}]=-0.85$ isochrone. Given the typical uncertainties in the abundance determinations, $\sigma_{[\mathrm{Fe} / \mathrm{H}]} \approx 0.08 \mathrm{dex}$, it appears that our isochrones correctly predict the location of the unevolved main sequence around $[\mathrm{Fe} / \mathrm{H}]=-0.85$.

Further support that our isochrones correctly predict the location of the unevolved main sequence comes from the following additional tests: (1) a comparison between single field stars with good parallaxes from Hipparcos and our isochrones found good agreement between $-1.5 \leq[\mathrm{Fe} / \mathrm{H}] \leq$ -1.0 (Chaboyer et al. 1998). This study was interested in metal-poor globular clusters, and so did 
not consider stars with $[\mathrm{Fe} / \mathrm{H}]>-1.0$. (2) With no adjustable parameters, our isochrones provide a good match to the main sequence of the Hyades $([\mathrm{Fe} / \mathrm{H}]=+0.15)$ derived from Hipparcos parallaxes (Chaboyer, Green \& Liebert 1999), and (3) we are using a solar calibrated mixing length which implies our models are in good agreement with main sequence solar metallicity stars. Clearly, these tests are not definitive as the number of single, metal-poor stars with well determined parallaxes is relatively small. However, these tests do show that the isochrones are not in gross disagreement with the observations, and suggest that at the level of $\approx \pm 0.10$, the isochrones correctly predict the location of the unevolved main sequence in relatively metal-poor stars $(-1.5 \leq[\mathrm{Fe} / \mathrm{H}] \leq 0.65)$.

\section{Analysis of the CMD}

The $(V, V-I)$ CMD for NGC 6652 obtained from the HST data is shown in Figure 4 . As discussed in $\S 2$, data from the long exposures $(V \geq 17)$ does not include stars with $r<11.5^{\prime \prime}$. The CMD clearly delineates the major evolutionary sequences from $\sim 4$ mag below the turn-off to $\sim 1.5$ mag above the HB. An inspection of the CMD leads to the following values: $V_{\mathrm{ZAHB}}=16.00 \pm 0.03$, $\left\langle V_{\mathrm{HB}}\right\rangle=15.96 \pm 0.04,(V-I)_{\mathrm{TO}}=0.818 \pm 0.004, V_{\mathrm{TO}}=19.55 \pm 0.07, V_{\mathrm{SGB}}=19.09 \pm 0.03$, $\Delta \mathrm{V}_{\mathrm{HB}}^{\mathrm{TO}}=3.59 \pm 0.08$, and $\Delta \mathrm{V}_{\mathrm{HB}}^{\mathrm{SGB}}=3.13 \pm 0.05$. All errors are estimated internal errors, which reflect the scatter of the photometry. The magnitude of the $\mathrm{HB}$ was estimated at the mid-point in the color of the $\mathrm{HB}$ (around $V-I \simeq 1.05$ ). The point $V_{\mathrm{SGB}}$ was defined by Chaboyer et al. (1996) to be the magnitude of the point on the sub-giant branch which is 0.05 mag redder than the turn-off. As discussed by Chaboyer et al. (1996) it is an excellent age indicator for old stellar systems. Recently, Ferraro et al. (1999) have presented an homogeneous catalog of HB parameters. They stress that due to the rapid evolution away from the zero-age horizontal branch (ZAHB), the observed lower envelope of the HB does not coincide with the ZAHB from theoretical models. The $V_{\mathrm{ZAHB}}$ level reported above is the lower envelope of the observations. Using their theoretical HB models, Ferraro et al. (1999) determined that a correction of $\simeq+0.04$ mag needs to be applied to the observed lower envelope of the NGC $6652 \mathrm{HB}$. If such a correction is necessary in our dataset then $V_{\mathrm{ZAHB}}=16.04 \pm 0.03$ using the methodology of Ferraro et al. (1999).

The $(V, V-I)$ CMD of OBB shows considerable scatter around the turn-off, so it is difficult to compare our photometry to that of $\mathrm{OBB}$ at the fainter magnitudes. However, it is possible to compare our photometry around the level of the HB. This comparison is shown in Figure 5. From this figure, it is clear that the level of the ZAHB is 0.03 mag fainter in the photometry of OBB as compared to the photometry presented in this paper. In addition, the color of the red giant branch (RGB) at the level of the HB as found by OBB is $0.08 \mathrm{mag}$ bluer than the RGB color in our photometry. The reason(s) for these differences is unclear. The differences are considerably larger than our estimated errors due to the aperture corrections, and are larger than the estimated errors in the HST $V, V-I$ calibration. To investigate this further, F439W and F555W (B,V) GO 6095 observations (Sosin et al. 1997) were retrieved from the HST archive and the data were reduced in exactly the same manner as was done with our $\mathrm{V}, V-I$ data. The results of this comparison are 
plotted in Figure 6. From this, one can see that the B-V RGBs are in better agreement between the two datasets than those in V-I. There is a slight offset in the level of the ZAHB, with the OBB photometry being brighter than the HST photometry. From the OBB $B-V$ data, we obtained $\left.<V_{\mathrm{HB}}\right\rangle=15.85$ and $(B-V)_{g}=1.11$ (color of the RGB at the level of the HB). From the VI data presented by $\mathrm{OBB}$, we find $\left\langle V_{\mathrm{HB}}\right\rangle=15.95$ and $(V-I)_{g}=1.11$. The disagreement between the level of the $\mathrm{HB}$ in the $B-V$ and $V-I$ datasets suggests that the OBB data is not internally consistent. In the $B-V$ and $V-I$ HST data, the level of the HB agrees to within $0.01 \mathrm{mag}$. In addition, OBB found $(B-V)_{g}=(V-I)_{g}$. In general, the $V-I$ color is redder than the $B-V$. color. Our isochrones show this trend, as does the observational transformation for globular cluster giant branches from $B-V$ to $V-I$ presented by Zinn \& Barnes (1996). From the HST data, we find $(B-V)_{g}=1.06$ and $(V-I)_{g}=1.22$. The Zinn \& Barnes (1996) transformation applied to the HST $B-V$ data implies $(V-I)_{g}=1.24$ in good agreement with the measured value.

Finally, we note that $\mathrm{OBB}$ determined a brighter turn-off magnitude, $V_{\mathrm{TO}} \approx 19.2 \pm 0.15$ and found $\Delta \mathrm{V}_{\mathrm{HB}}^{\mathrm{TO}} \approx 3.35 \pm 0.16$. These values differ by $2.5 \sigma$ and $2.1 \sigma$ respectively from the values obtained with our photometry. As the turn-off magnitude is a primary age indicator, the fainter turn-off found in our photometry implies an older age for NGC 6652.

\section{The Age of NGC 6652}

\subsection{Isochrone Fitting}

A variety of methods may be used to determine the age of a globular cluster, each with their advantages and disadvantages. Perhaps the simplest method is to fit an isochrone to the data by adjusting the distance modulus and reddening (within their known uncertainties) such that the mean locus of observed points is matched to the theoretical isochrone. Due to uncertainties in surface boundary conditions, color-effective temperature transformations and the treatment of convection, the colors of the isochrones may be in error. This is particularly true on the RGB, where a modest change in the choice of the mixing length can lead to a large change in the predicted RGB colors. For this reason, when fitting the isochrones to the data only the main sequence and turn-off regions were used in the fit. Such a fit is shown in Figure 7 for our $[\mathrm{Fe} / \mathrm{H}]=-0.85$, $[\alpha / \mathrm{Fe}]=+0.4$ isochrones. The best fitting isochrone has an age of $13 \mathrm{Gyr}$. The best fit distance modulus $\left((m-M)_{V}=15.15\right)$ implies $M_{V}(H B)=0.81 \pm 0.04$. This is in good agreement with the distance modulus derived using the preferred $\mathrm{M}_{\mathrm{v}}(\mathrm{RR})$ relation given by Chaboyer (1999) which implies $M_{V}(H B)=0.73 \pm 0.12$ at the metallicity of NGC 6652. The best fit reddening of $\mathrm{E}(V-I)=$ 0.15 corresponds to $\mathrm{E}(B-V)=0.12$ and is in reasonable agreement with the value obtained by OBB $(\mathrm{E}(B-V)=0.10 \pm 0.02)$. The Schlegel, Finkbeiner \& Davis (1998) reddening maps based on IRAS/DIRBE dust emission yield $\mathrm{E}(B-V)=0.114$ for NGC 6652, also in good agreement.

When performing main sequence fitting, there is considerable degeneracy between the derived distance modulus and the reddening. For example, changing the reddening to $\mathrm{E}(V-I)=0.13$, 
leads to a derived distance modulus of $(m-M)_{V}=15.05$ and an age of $15 \mathrm{Gyr}$. Assuming an uncertainty of the reddening of $\pm 0.02 \mathrm{mag}$ leads to an uncertainty in the derived distance modulus of $\pm 0.10 \mathrm{mag}$ and in the age of $\pm 2 \mathrm{Gyr}$. In fitting the isochrones to the data, the degeneracy between the distance modulus and reddening was broken by examining the region around the turnoff and the sub-giant branch to obtain the best fit shown in Figure 7. This allows us to determine our preferred values for the age and distance modulus, but it is important to realize that the error in these best fit parameters are rather large.

The implicit assumption in this fitting procedure is that the location of the unevolved main sequence predicted by the theoretical isochrones is accurate. The validity of this assumption has been discussed at the end of $\S 3$, where we conclude that at the level of $\approx \pm 0.10$ mag the isochrones correctly predict the location of the unevolved main sequence in relatively metal-poor stars $(-1.5 \leq$ $[\mathrm{Fe} / \mathrm{H}] \leq 0.6)$. Combining the uncertainty in the theoretical location of the unevolved main sequence with the uncertainty in our fitting procedure due to errors in the reddening leads us to conclude that the age and distance modulus we derive from isochrone fitting are accurate to $\pm 15 \%$ and \pm 0.15 mag respectively.

Fits of the isochrones with the various compositions discussed in $§ 3$ were also performed. In all cases, the fits in the turn-off, sub-giant branch and RGB regions were considerably inferior to the fit shown in Figure 7 which uses our best estimate for the composition $([\mathrm{Fe} / \mathrm{H}]=-0.85$; $[\alpha / \mathrm{Fe}]=+0.4)$. The best fitting parameters for the various isochrone fits are shown in Table 2 . Given that the other compositions gave inferior fits to the data, we prefer to take as our central

values those found from the isochrones with the best estimate for the compositions. Taking into account the uncertainty in the reddening, along with the variation in derived parameters shown in Table 2, isochrone fitting implies the following parameters for NGC $6652(m-M)_{V}=15.15 \pm 0.15$, $\mathrm{E}(V-I)=0.15 \pm 0.02$ and an age of $13 \pm 2 \mathrm{Gyr}$. Due to uncertainties in the theoretical models and the reddening of NGC 6652, the age we derive based upon isochrone fitting has a relatively large error. The isochrone fitting procedure serves as a first order test of our models (ensuring they are in reasonable agreement with the observations) and allows us to determine the age of the cluster which is independent of the HB.

\section{2. $\Delta V_{\mathrm{HB}}^{\mathrm{SGB}}$ Ages}

A more robust determination of the cluster's age may be found using the difference in magnitude of the point on the sub-giant branch which is 0.05 mag redder than the turn-off and the $\mathrm{HB}$ (Chaboyer et al. 1996, referred to as $\Delta V_{\mathrm{HB}}^{\mathrm{SGB}}$ ). The magnitude of the SGB point as a function of age is determined from the isochrones. The theoretical value of $M_{\mathrm{V}}(\mathrm{HB})$ was calculated using $\mathrm{M}_{\mathrm{v}}(\mathrm{RR})=0.23 *([\mathrm{Fe} / \mathrm{H}]+1.6)+0.56$ (Chaboyer 1999) and corrected for the fact that the HB was only apparent redward of the RR Lyrae instability strip in NGC 6652. This correction was determined using the theoretical HB models of Demarque et al. (2000). From this procedure, we obtain an age of $11.7 \pm 1.6 \mathrm{Gyr}$, where the error includes a \pm 0.1 dex uncertainty in $[\mathrm{Fe} / \mathrm{H}]$, the 
observational error in determining $\Delta V_{\mathrm{HB}}^{\mathrm{SGB}}$, and the error in our adopted $\mathrm{M}_{\mathrm{v}}(\mathrm{RR})$ calibration. This may be compared to an age of $13 \pm 2 \mathrm{Gyr}$ which was found from isochrone fitting. This age difference of $1.3 \pm 2.6 \mathrm{Gyr}$ is well within our errors. From the theoretical point of view, $\Delta \mathrm{V}_{\mathrm{HB}}^{\mathrm{SGB}}$ is a more robust age indicator than isochrone fitting as it does not depend critically on the (uncertain) colors of the theoretical models (Chaboyer et al. 1996). Thus, we prefer the use of the $\Delta V_{\mathrm{HB}}^{\mathrm{SGB}}$ age indicator. Ignoring the error in the zero-point calibration of our $M_{v}(R R)$ relation gives a precise relative age for NGC 6652 of $11.7 \pm 1.0 \mathrm{Gyr}$.

The above relative age may be compared to other clusters with a similar metallicity. For example, there exists excellent $V-I$ photometry of the thick disk globular cluster 47 Tuc from Kaluzny et al. (1998). This cluster has a well established metallicity of $[\mathrm{Fe} / \mathrm{H}]=-0.71$ on both the Zinn \& West (1984) and Carretta \& Gratton (1997) scales, which is 0.14 dex more metal-rich than our preferred $[\mathrm{Fe} / \mathrm{H}]$ for NGC 6652 . This difference in metallicity precludes the use of the $\delta$-color technique to determine an age difference for the two clusters.

The electronic data of Kaluzny et al. (1998) was used to determine $(V-I)_{\mathrm{TO}}=0.714 \pm 0.004$, $V_{\mathrm{SGB}}=17.25 \pm 0.03,\left\langle V_{\mathrm{HB}}\right\rangle=14.07 \pm 0.04, \Delta \mathrm{V}_{\mathrm{HB}}^{\mathrm{SGB}}=3.18 \pm 0.05$. These values were determined in exactly the same manner as for NGC 6652. The value of $\Delta V_{\mathrm{HB}}^{\mathrm{SGB}}$ for 47 Tuc is very similar to that of NGC 6652. Formally, $\Delta \mathrm{V}_{\mathrm{HB}}^{\mathrm{SGB}}(\mathrm{N} 6652-47 \mathrm{Tuc})=-0.05 \pm 0.07$. Assuming that 47 Tuc has $[\alpha / \mathrm{Fe}]=+0.40$ and a helium abundance which is given by eqn. (1) then a precise relative age of $12.2 \pm 0.7 \mathrm{Gyr}$ is obtained for 47 Tuc using the $\Delta \mathrm{V}_{\mathrm{HB}}^{\mathrm{SGB}}$ method. This is virtually identical to the age derived for NGC 6652. To the precision of the data, the two clusters have the same age to within $\pm 1.2 \mathrm{Gyr}$.

Brown \& Wallerstein (1992) found an enhancement in $\alpha$-capture elements of $[\alpha / \mathrm{Fe}]=0.22$ in 47 Tuc. Using this value of $[\alpha / \mathrm{Fe}]$ and using the same assumptions as this paper, Liu \& Chaboyer (2000) found a $\Delta V_{\mathrm{HB}}^{\mathrm{SGB}}$ age of $12.9 \pm 0.7 \mathrm{Gyr}$ for 47 Tuc implying that 47 Tuc is $1.2 \pm 1.2 \mathrm{Gyr}$ older than NGC 6652. If 47 Tuc has a solar helium abundance, the absolute magnitude of the HB would be different than what has been assumed, implying that the derived age would be altered. Salaris \& Weiss (1998) found that increasing the helium abundance by 0.019 to (their) solar value decreased the estimated age of 47 Tuc by $1.1 \mathrm{Gyr}$. This difference in helium abundance between our solar value and that implied by eqn. (1) is 0.0177 , and so if 47 Tuc has a solar helium abundance the $\triangle \mathrm{V}_{\mathrm{HB}}^{\mathrm{SGB}}$ age we determined should be decreased by $1.0 \mathrm{Gyr}$. This would imply that the NGC 6652 is $0.9 \pm 1.2 \mathrm{Gyr}$ older than 47 Tuc. Thus, allowing for differences in the $[\alpha / \mathrm{Fe}]$ or in the helium abundance between the two clusters does not change our conclusion that, to the precision of the data, NGC 6652 and 47 Tuc are the same age. This lack of age difference between 47 Tuc and NGC 6652 implies that the formation of the thick disk overlapped with the formation of the inner halo.

At the suggestions of the referee, we have tested the results of our $\Delta \mathrm{V}_{\mathrm{HB}}^{\mathrm{SGB}}$ analysis by overlaying the CMD of 47 Tuc with that of NGC 6652. The 47 Tuc data was shifted so that the turnoff colors and the luminosities of the observed ZAHBs are made coincident. The results of this comparison are shown in Figure 8. A careful inspection of this figure reveals that when the ZAHBs of the 2 
clusters are made coincident, the 47 Tuc subgiant branch is slightly fainter (by $0.06 \pm 0.03 \mathrm{mag}$ ) than the subgiant branch of NGC 6652 . Thus, $\Delta \mathrm{V}_{\mathrm{HB}}^{\mathrm{SGB}}$ for 47 Tuc is slightly larger than $\Delta \mathrm{V}_{\mathrm{HB}}^{\mathrm{SGB}}$ in NGC 6652 . This is exactly what was determined by the $\Delta \mathrm{V}_{\mathrm{HB}}^{\mathrm{SGB}}$ analysis presented in the previous paragraph.

The age of NGC 6652 may also be compared to NGC 6171 (M107). M107 is also considered a member of the halo (Zinn 1985). From their Ca II triplet analysis, Rutledge, Hesser \& Stetson (1997) found $[\mathrm{Fe} / \mathrm{H}]=-1.09$ on the Zinn \& West (1984) scale and $[\mathrm{Fe} / \mathrm{H}]=-0.95$ on the Carretta \& Gratton (1997) scale. This implies that M107 is $\simeq 0.1$ dex more metal-poor than NGC 6652 . A V,$B-V$ CMD for M107 has been presented by Ferraro et al. (1991). As no V, $V-I$ data is publicly available for this cluster, we cannot do a differential age comparison using the $\delta$-color technique, and instead analyze the age difference using $\Delta \mathrm{V}_{\mathrm{HB}}^{\mathrm{SGB}}$. The electronic data of Ferraro et al. (1991) was carefully inspected, and the following points measured: $(B-V)_{\text {To }}=0.870 \pm 0.01$, $V_{\mathrm{SGB}}=18.86 \pm 0.04,\left\langle V_{\mathrm{HB}}>=15.59 \pm 0.03, \Delta V_{\mathrm{HB}}^{\mathrm{SGB}}=3.27 \pm 0.05\right.$. This value of $\Delta V_{\mathrm{HB}}^{\mathrm{SGB}}$ is $0.14 \pm 0.07 \mathrm{mag}$ larger than that found for NGC $6652\left(\Delta \mathrm{V}_{\mathrm{HB}}^{\mathrm{SGB}}=3.13 \pm 0.05\right)$. Using our isochrones, an $\Delta \mathrm{V}_{\mathrm{HB}}^{\mathrm{SGB}}$ age for M107 of $14.0 \pm 1.1 \mathrm{Gyr}$ is found, which is $2.7 \pm 1.5 \mathrm{Gyr}$ older than the $\Delta V_{\mathrm{HB}}^{\mathrm{SGB}}$ age of NGC 6652 . This suggests that M107 may be older than NGC 6652 . However, this result is only significant at the $1.8 \sigma$ level. A more definitive study (using $V-I$ photometry for M107) is needed to determine if NGC 6652 is younger than M107.

To investigate this issue further, we have also determined the age of NGC 1851, a somewhat more metal-poor cluster. Rutledge, Hesser \& Stetson (1997) found $[\mathrm{Fe} / \mathrm{H}]=-1.23$ on the Zinn \& West (1984) scale and $[\mathrm{Fe} / \mathrm{H}]=-1.03$ on the Carretta \& Gratton (1997) scale, implying that NGC 1851 is $\simeq 0.2$ dex more metal-poor than NGC 6652. Walker (1998) obtained BVI CCD data for this cluster. Using the $V, V-I$ data, we determined $(V-I)_{\text {TO }}=0.581 \pm 0.01, V_{\mathrm{SGB}}=19.11 \pm 0.04$, $\left\langle V_{\mathrm{HB}}\right\rangle=16.12 \pm 0.04, \Delta \mathrm{V}_{\mathrm{HB}}^{\mathrm{SGB}}=2.99 \pm 0.06$. This implies an age of $10.4 \pm 1.0 \mathrm{Gyr}$, adopting $[\mathrm{Fe} / \mathrm{H}]=-1.10$. Thus, NGC 1851 is $0.9 \pm 1.4 \mathrm{Gyr}$ younger than $\mathrm{NGC} 6652$, and $3.6 \pm 1.5 \mathrm{Gyr}$ younger than M107. Salaris \& Weiss (1998) and Rosenberg et al. (1999) have also concluded that NGC 1851 is somewhat younger than M107 $(2.1 \pm 1.4 \mathrm{Gyr}$ and $2.9 \pm 1.3 \mathrm{Gyr}$ respectively, using different age diagnostics). In summary, there is a suggestion that NGC 6652 is somewhat younger $(2.7 \pm 1.5 \mathrm{Gyr})$ than the halo cluster M107, while it appears to be slightly older $(0.9 \pm 1.4 \mathrm{Gyr})$ than NGC 1851.

\section{Summary}

HST $(V, I)$ photometry has been obtained for the inner halo globular cluster NGC 6652. This photometry includes a well populated $\mathrm{HB}$ and extends well below the main sequence turn-off. From these data, we determined $V_{\mathrm{ZAHB}}=16.00 \pm 0.03,\left\langle V_{\mathrm{HB}}\right\rangle=15.96 \pm 0.04,(V-I)_{\mathrm{TO}}=0.818 \pm 0.004$,

$V_{\mathrm{TO}}=19.55 \pm 0.07, V_{\mathrm{SGB}}=19.09 \pm 0.03, \Delta \mathrm{V}_{\mathrm{HB}}^{\mathrm{TO}}=3.59 \pm 0.08$, and $\Delta \mathrm{V}_{\mathrm{HB}}^{\mathrm{SGB}}=3.13 \pm 0.05$. This turn-off magnitude is $0.35 \pm 0.17$ magnitudes fainter than that found in the previous study of NGC 6652 by OBB. As a consequence, the age derived from our photometry is substantially older than 
age determinations based upon the OBB photometry. The OBB photometry shows considerable scatter around the turn-off, while the data presented here clearly delineates the main sequence turn-off region.

New stellar evolution models and isochrones were calculated for $[\mathrm{Fe} / \mathrm{H}]=-1.20,-1.00,-0.85$ and -0.70 in order to determine the age of NGC 6652 and other globular clusters with similar metallicities. Estimates for the metallicity of NGC 6652 vary from $[\mathrm{Fe} / \mathrm{H}]=-0.96$ on the Zinn \& West (1984) scale to $[\mathrm{Fe} / \mathrm{H}]=-0.85$ on the Carretta \& Gratton (1997) scale. Our best fitting isochrones have $[\mathrm{Fe} / \mathrm{H}]=-0.85$ and imply $(m-M)_{V}=15.15 \pm 0.10$ and $\mathrm{E}(V-I)=0.15 \pm 0.02$. With $\mathrm{R}_{\mathrm{GC}} \simeq 2.0 \mathrm{kpc}$, NGC 6652 is the globular cluster closest to the Galactic center for which a precise relative age comparison can be made to other globular clusters which are relatively metalpoor. From the HST data, NGC 6652 has an age of $11.7 \pm 1.6 \mathrm{Gyr}$ (using $\Delta \mathrm{V}_{\mathrm{HB}}^{\mathrm{SGB}}$ as the age indicator). Using data from the literature, $\Delta \mathrm{V}_{\mathrm{HB}}^{\mathrm{SGB}}$ ages of $12.2 \pm 0.7 \mathrm{Gyr}$ for 47 Tuc (thick disk cluster with $[\mathrm{Fe} / \mathrm{H}]=-0.71$ ) $14.0 \pm 1.1 \mathrm{Gyr}$ for M107 (halo cluster with $[\mathrm{Fe} / \mathrm{H}]=-0.95$ ) and $10.4 \pm 1.0 \mathrm{Gyr}$ for NGC 1851 (halo cluster with $[\mathrm{Fe} / \mathrm{H}] \simeq-1.1$ ) were determined. These precise relative ages demonstrate that the halo clusters NGC 6652 and NGC 1851 are the same age as the thick disk cluster 47 Tuc. There is some evidence that M107 is somewhat older, but the difference in age between M107 and NGC 6652 is only significant at the $1.5 \sigma$ level. A more definitive study (using $V-I$ photometry for M107) is needed to determine if NGC 6652 is younger than M107.

We would like to thank the anonymous referee whose suggestions led to an improved paper. This research was supported by NASA through grant number GO-06517 from the Space Telescope Science Institute, which is operated by AURA, Inc., under NASA contract NAS 5-26555.

\section{REFERENCES}

Bahcall, J.N. 1989, Neutrino Astrophysics (Cambridge: Cambridge Univ. Press)

Bahcall, J.N., \& Pinsonneault, M.H. 1992, Rev. Mod. Phys. 64, 885

Bessell, M.S., Castelli, F., \& Plez, B. 1998, A\&A, 333, 231

Brown, J. A. \& Wallerstein, G. 1992, AJ, 104, 1818

Carretta, E. \& Gratton, R.G. 1997, A\&AS, 121, 95

Carretta, E., Gratton, R. G. and Sneden, C. 2000, A\&A, 356, 238

Cayrel de Strobel, G., Soubiran, C., Friel, E.D., Ralite, N. \& Francois, P. 1997, A\&AS, 124, 299

Chaboyer, B. 1999, Post-Hipparcos Cosmic Candles, ed. by A. Heck and F. Caputo (Dordrecht: Kluwer), 111 
Chaboyer, B., Demarque, P., Kernan, P. J., Krauss, L. M. \& Sarajedini, A. 1996, MNRAS, 283, 683

Chaboyer, B. , Demarque, P., Kernan, P. J. \& Krauss, L. M. 1998, ApJ, 494, 96

Chaboyer, B. , Green, E. M. \& Liebert, J. 1999, AJ, 117, 1360

Chaboyer, B., \& Kim, Y.-C. 1995, ApJ, 454, 767

Clementini, G., Gratton, R. G., Carretta, E. \& Sneden, C. 1999, MNRAS, 302, 22

Demarque, P., Zinn, R., Lee, Y. \& Yi, S. 2000, AJ, 119, 1398

Dorman, B., Vandenberg, D. A. and Laskarides, P. G. 1989, ApJ, 343, 750

Ferraro, F. R., Clementini, G., Fusi Pecci, F. \& Buonanno, R. 1991, MNRAS, 252, 357

Ferraro, F.R., Messineo, M., Fusi Pecci, F., De Palo, M.A., Straniero, O, Chieff, A. \& Limongi, M. 1999, AJ, 118, 1738

Grevesse, N., \& Noels, A. 1993, in Origin and Evolution of the Elements, ed. N. Prantzos, E. Vangioni-Flam \& M. Casse (Cambridge: Cambridge Univ. Press), 15

Guenther, D.B., Demarque, P., Kim, Y.-C., \& Pinsonneault, M.H. 1992, ApJ, 387, 372

Harris, W.E. 1996, AJ, 112, 1487

Holtzman, J. A. et al. 1995, PASP, 107, 1065

Iglesias, C.A., \& Rogers, F. 1996, ApJ, 464, 943

Kaluzny, J., Wysocka, A., Stanek, K. Z. \& Krzeminski, W. 1998, Acta Astronomica, 48, 439

Kurucz, R.L. 1991, in Stellar Atmospheres: Beyond Classical Models, ed. L. Crivellari, I. Hubeny, D.G. Hummer, (Dordrecht: Kluwer) 440

Kurucz, R.L. 1993, CD-ROM No. 13 (Cambridge: Smithsonian Astrophysical Observatory)

Lambert, D.L. 1989, in Cosmic Abundances of Matter, AIP conference proceedings 183, ed. C.J. Waddington (New York:American Institute of Physics) 168

Liu, W.M. \& Chaboyer, B. 2000, ApJ, in press

Michaud, G., \& Proffitt, C.R. 1993, in Inside the Stars, IAU Coll. 137, ed. W.W. Weiss \& A. Baglin (San Francisco: PASP), 246

Olive, K., Steigman, G., \& Skillman, E.D. 1997, ApJ, 483, 788

Ortolani, S., Bica, E. \& Barbuy, B. 1994, A\&A, 286, 444 (OBB) 
Prather, M. 1976, Ph.D. thesis, Yale University

Reid, N. 1998, AJ, 115, 204

Rogers, F.J. 1994, in The Equation of State in Astrophysics, IAU Coll. 147, ed. G. Chabrier \& E. Schatzman (Cambridge: Cambridge University Press), 16

Rosenberg, A., Saviane, I., Piotto, G. \& Aparicio, A. 1999, AJ, 118, 2306

Rutledge, G.A., Hesser, J.E. \& Stetson, P.B. 1997, PASP, 109, 907

Sosin, C., Piotto, G., Djorgovski, S.G., King, I.R., Rich, R.M., Dorman, B., Liebert, J., \& Renzini, A. 1997, in Advances in Stellar Evolution, Ed. by R.T. Rood \& A. Renzini (Cambridge: Cambridge University Press), 92

Salaris, M. \& Weiss, A. 1998, A\&A, 335, 943

Schlegel, D.J., Finkbeiner, D.P. \& Davis, M. 1998, ApJ, 500, 525

Stetson, P. B. 1987, PASP, 99, 191

Stetson, P. B. 1994, PASP, 106, 250

Tomkin, J. \& Lambert, D.L., 1999, ApJ, 523, 234

Weiss, A. \& Salaris, M. 1999, A\&A, 346, 897

Walker, A. R. 1998, AJ, 116, 220

Whitmore, B., Heyer, I., \& Casertano, S. 1999, PASP, 111, 1559

Zinn, R. 1985, ApJ, 293, 424

Zinn, R. 1993, in The Globular Cluster-Galaxy Connection, eds. G.H. Smith \& J.P. Brodie (San Francisco: ASP), 38

Zinn, R. \& Barnes, S. 1996, AJ, 112, 1054

Zinn, R. \& West, M. 1984, ApJS, 55, 45 
Table 1. Photometric Data

\begin{tabular}{rrrrc}
\hline \hline Chip & X (pixel) & Y (pixel) & V (mag) & $V-I$ (mag) \\
\hline PC1 & 420.61 & 462.47 & 14.698 & 1.214 \\
PC1 & 665.83 & 481.91 & 14.852 & 1.434 \\
PC1 & 731.93 & 164.93 & 14.929 & 1.362 \\
PC1 & 415.13 & 514.46 & 14.969 & 1.347 \\
PC1 & 290.54 & 521.16 & 15.071 & 1.299 \\
PC1 & 277.56 & 709.88 & 15.174 & 1.322 \\
PC1 & 250.74 & 585.43 & 15.413 & 1.303 \\
PC1 & 382.62 & 258.89 & 15.489 & 1.256 \\
PC1 & 487.84 & 607.94 & 15.496 & 1.083 \\
PC1 & 390.07 & 525.26 & 15.560 & 1.260 \\
PC1 & 639.05 & 254.91 & 15.623 & 1.245 \\
PC1 & 269.85 & 435.04 & 15.651 & 1.102 \\
PC1 & 446.00 & 487.11 & 15.659 & 1.267 \\
\hline
\end{tabular}

Table 2. Isochrone Fit Parameters

\begin{tabular}{ccccccc}
\hline \hline$[\mathrm{Fe} / \mathrm{H}]$ & {$[\alpha / \mathrm{Fe}]$} & $Y$ & $(m-M)_{V}$ & $\mathrm{E}(V-I)$ & Age (Gyr) & Note \\
\hline-0.85 & 0.40 & 0.2420 & 15.15 & 0.15 & 13 & best fit \\
-0.85 & 0.40 & 0.2420 & 15.05 & 0.13 & 15 & good fit \\
-0.85 & 0.00 & 0.2379 & 15.09 & 0.17 & 14 & poor fit to RGB \\
-1.00 & 0.40 & 0.2395 & 15.13 & 0.16 & 14 & poor fit to SGB \\
-0.70 & 0.40 & 0.2453 & 15.12 & 0.12 & 13 & poor fit to RGB \\
-0.70 & 0.40 & 0.2630 & 15.12 & 0.12 & 13 & poor fit to RGB \\
\hline
\end{tabular}




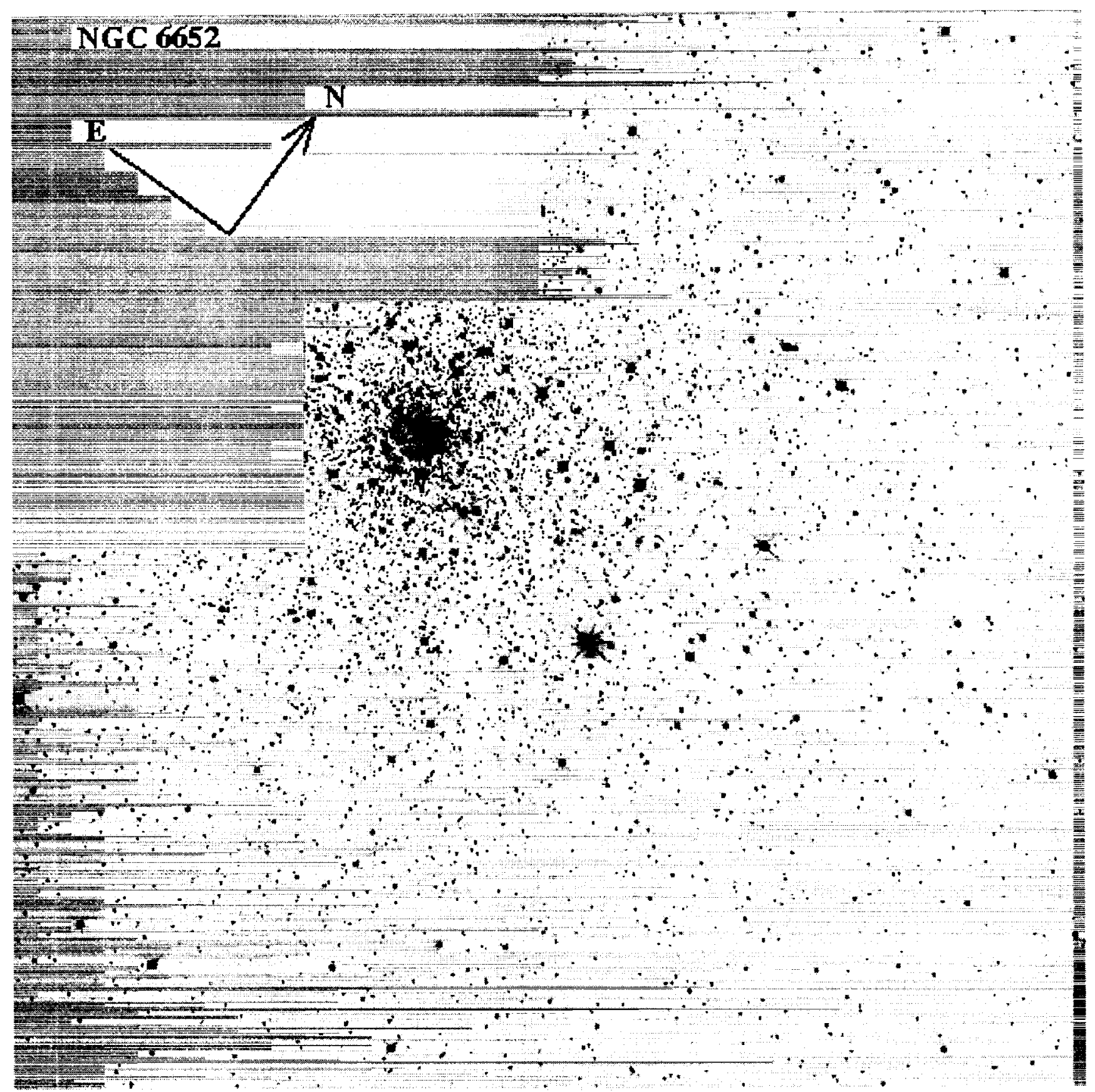

Fig. 1.- The averaged long exposure $V$ frame of NGC 6652 . 

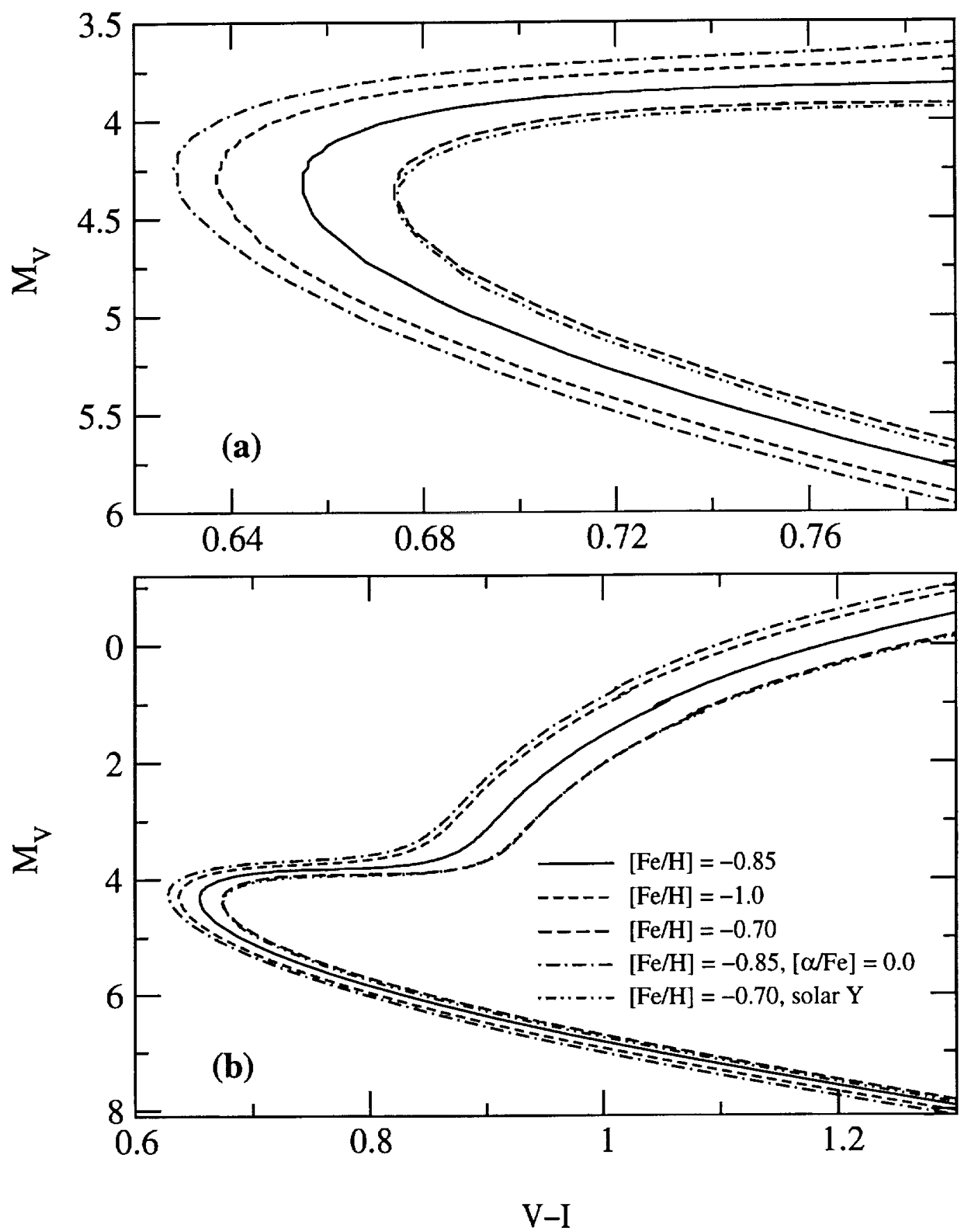

Fig. 2.- The $\left(M_{V}, V-I\right) \mathrm{CMD}$ of the $12 \mathrm{Gyr}$ theoretical isochrones used in this paper. Unless otherwise noted in the legend, the isochrones have $[\alpha / \mathrm{Fe}]=+0.4$ and a helium mass fraction $Y$ given by equation (1). The lower panel (b) shows the isochrones over the entire range of validity of the color transformation $(V-I \leq 1.3)$, while the upper panel (a) is an expanded view near the turn-off. 


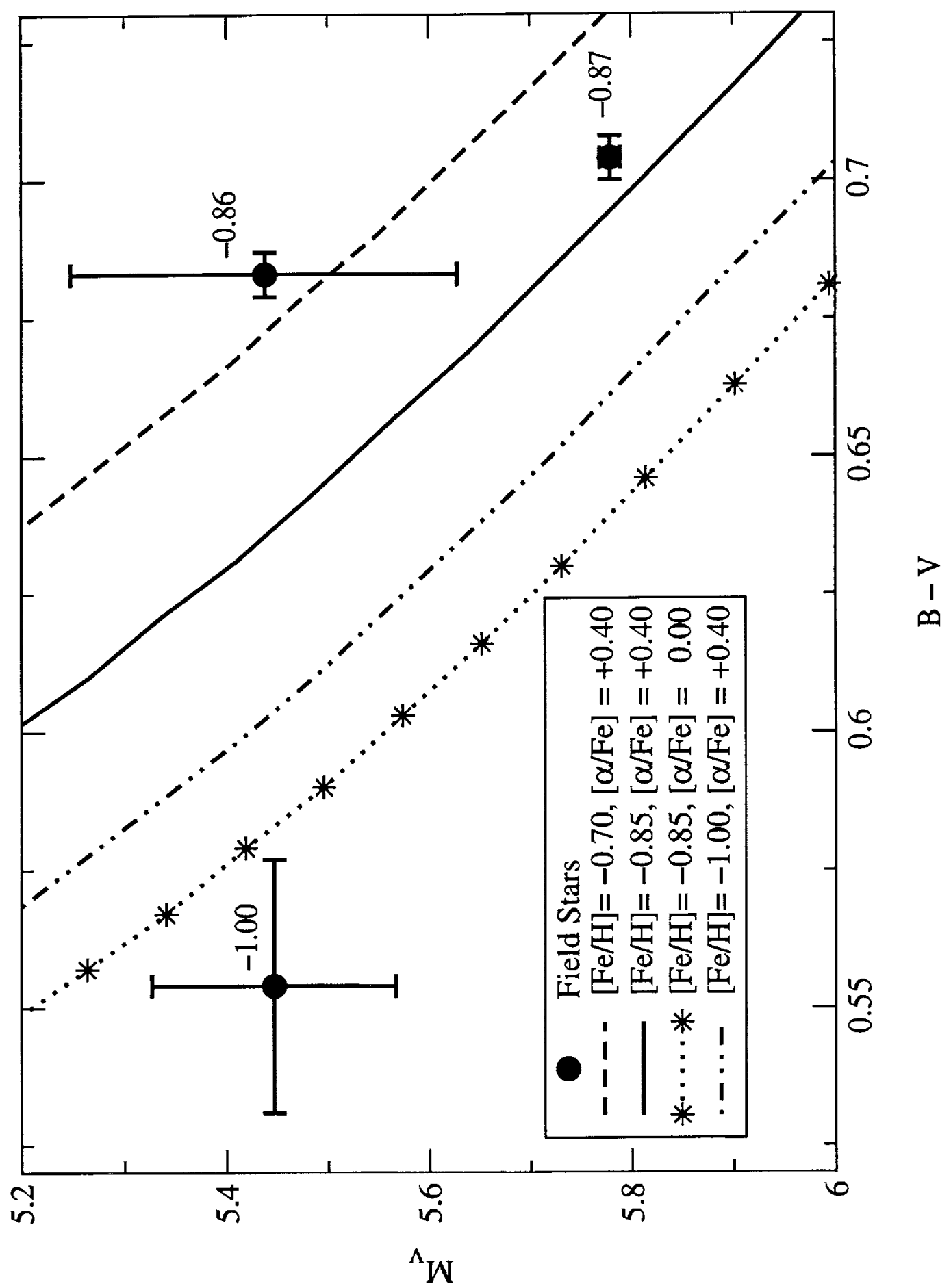

Fig. 3.- A comparison between unevolved, single stars in the Hipparcos catalogue (with $-1.05 \leq$ $[\mathrm{Fe} / \mathrm{H}] \leq-0.65$ and good parallaxes, $\left.\sigma_{\pi} / \pi<0.10\right)$ with the isochrones presented in this paper. Each of the stars (HD 6582, 193901, 216179) has been labeled with its [Fe/H] value determined from high dispersion spectroscopy. 


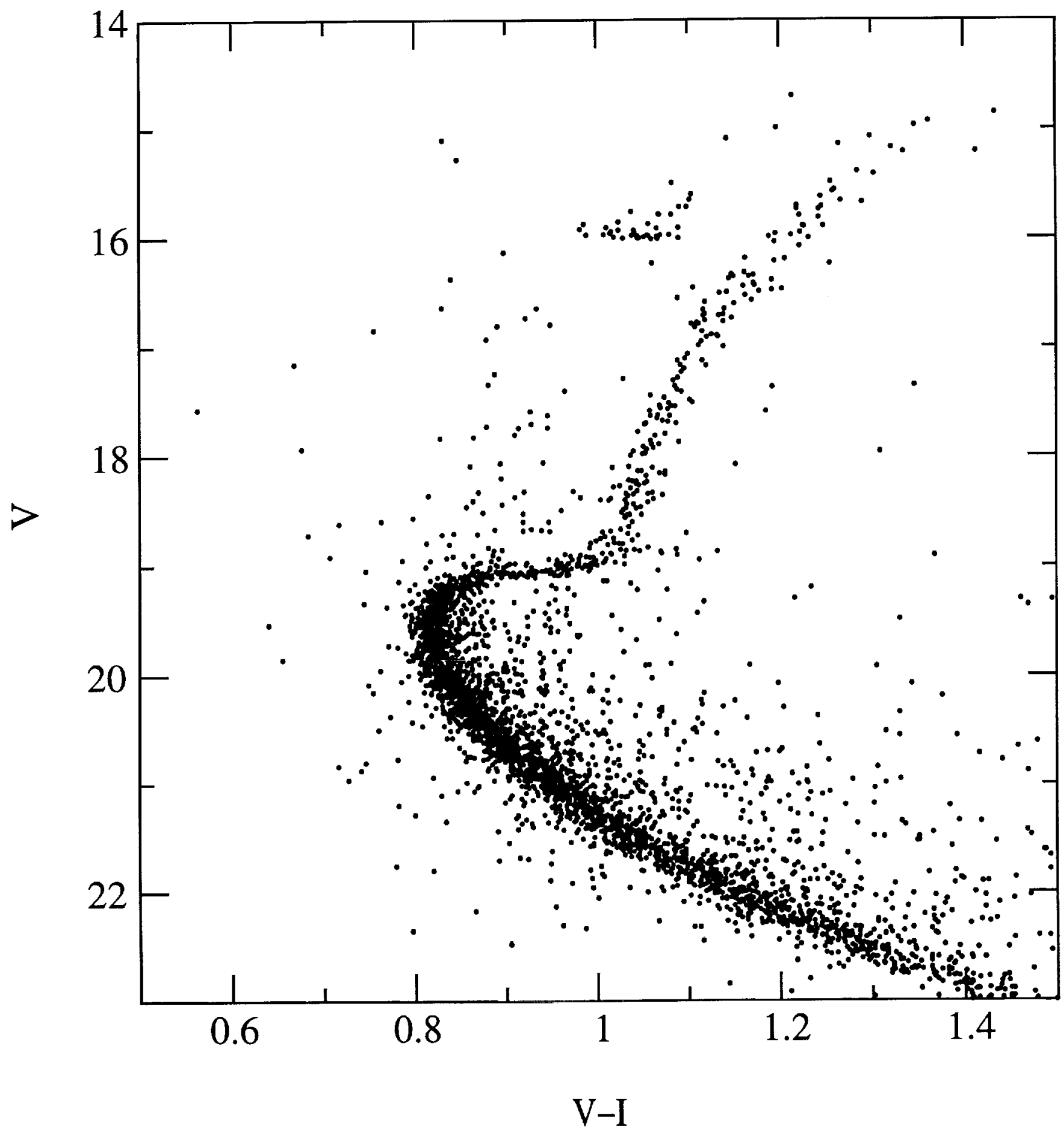

Fig. 4.- The $(V, V-I)$ CMD of NGC 6652. 


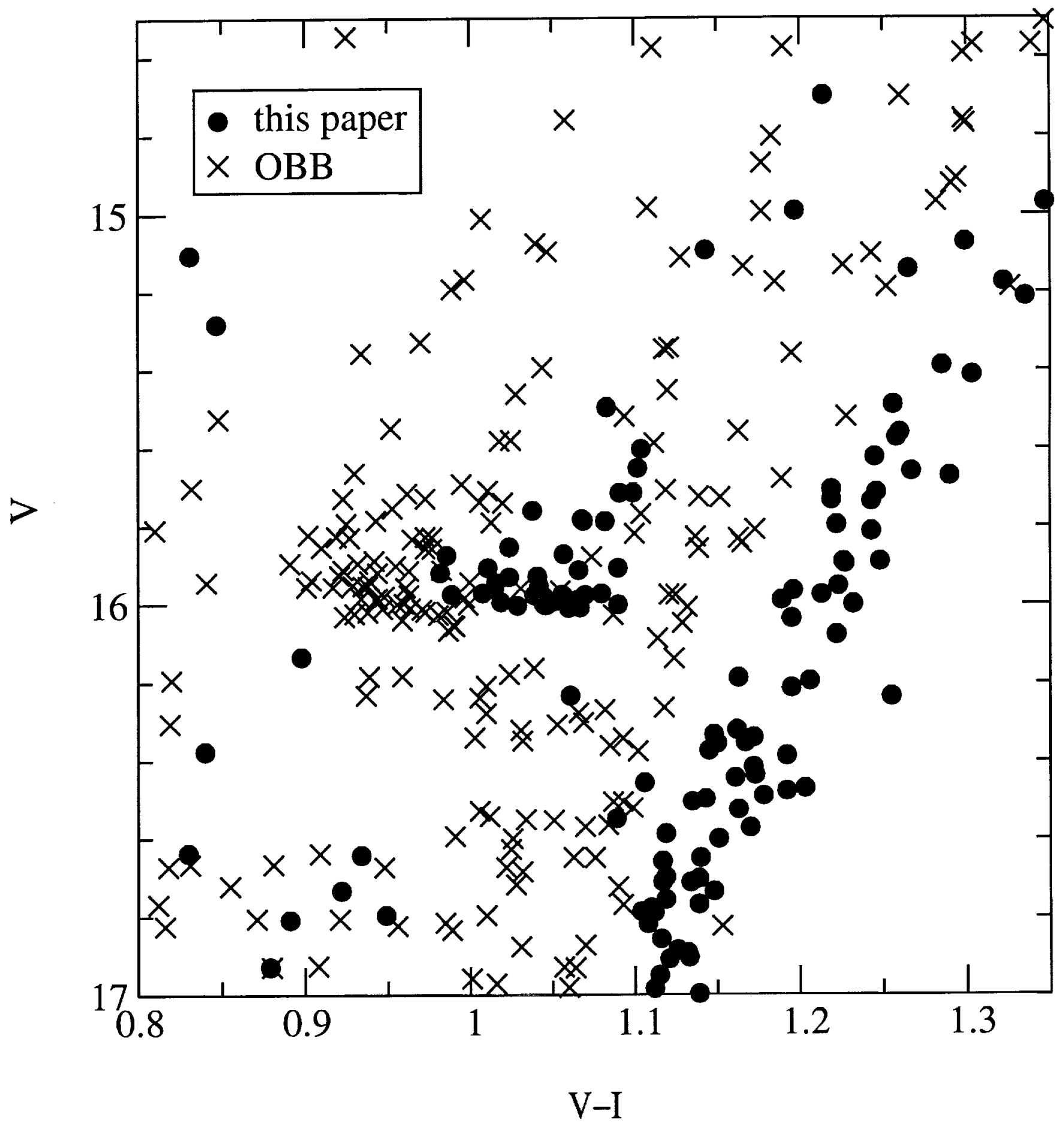

Fig. 5.- A comparison of the photometry near the HB presented in this paper, to that presented by OBB. 


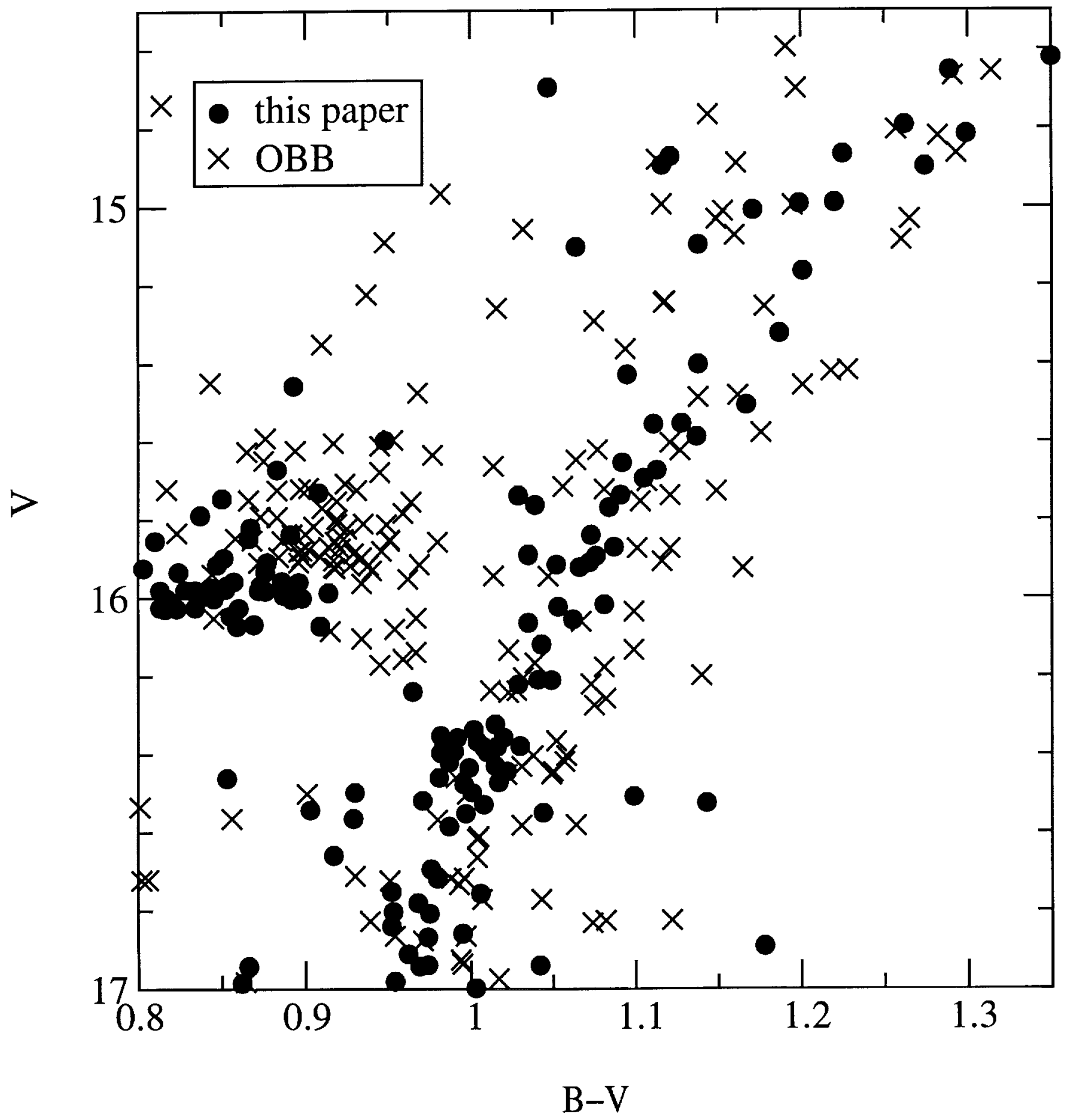

Fig. 6.- A comparison of the V, $B-V$ photometry measured from archival HST/WFPC2 observations of NGC 6652 to that presented by OBB in the region of the HB. 


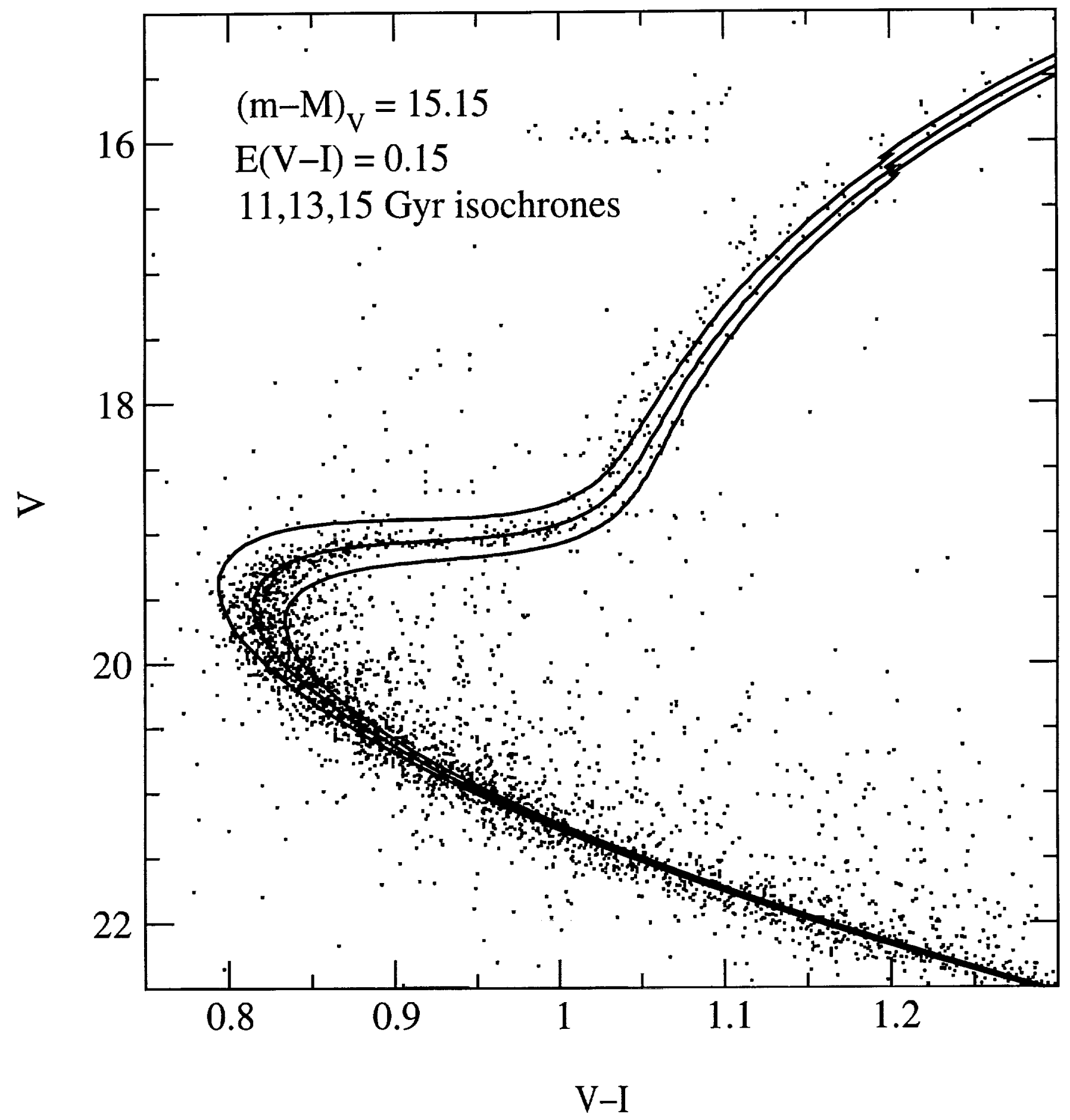

Fig. 7. - The fit of the $[\mathrm{Fe} / \mathrm{H}]=-0.85,[\alpha / \mathrm{Fe}]=+0.4$ isochrones to the data. 


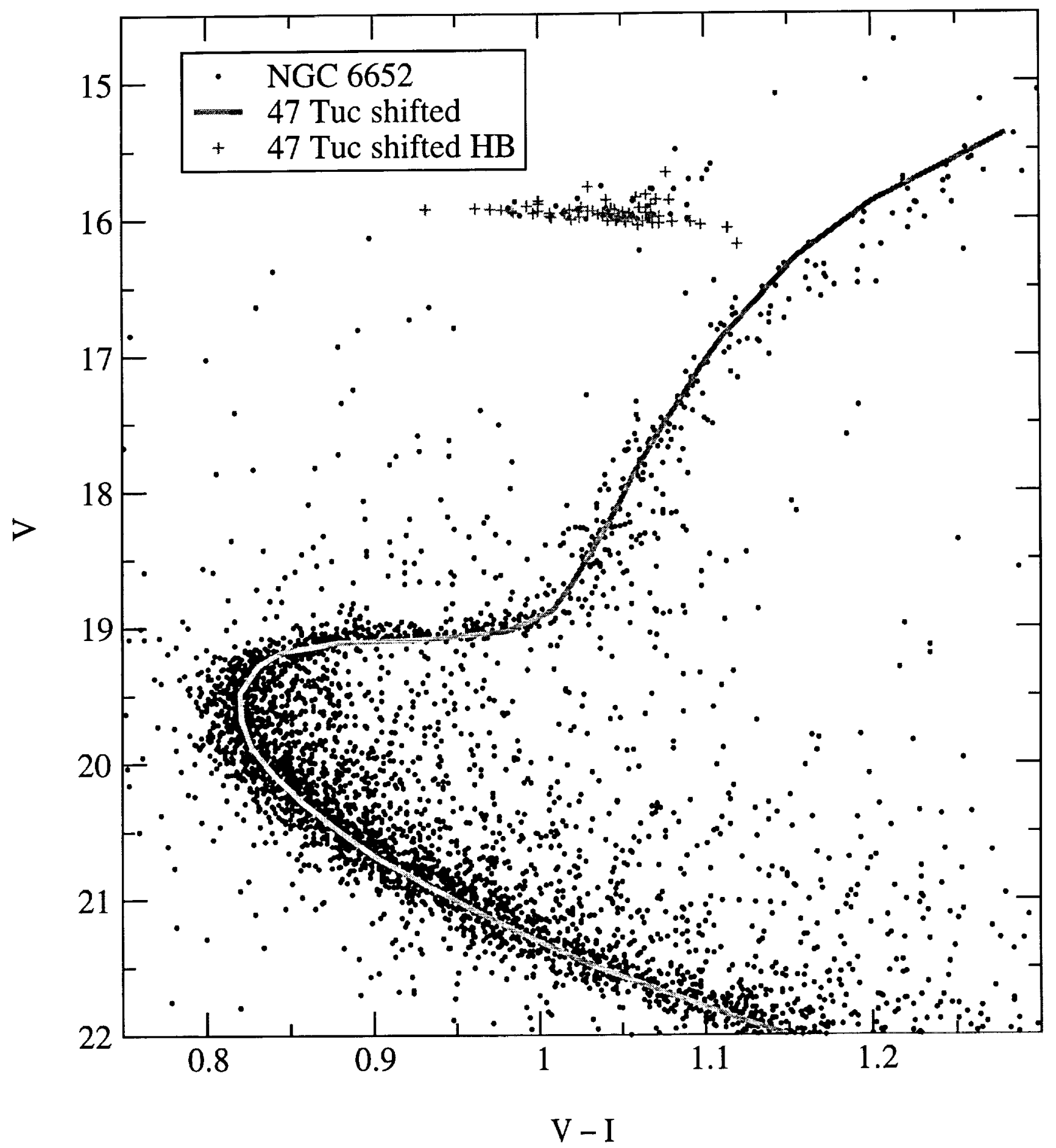

Fig. 8.- A comparison between our data for NGC 6652 and the Kaluzny et al. (1998) data for 47 Tuc. To facilitate the comparison, a fiducial for the 47 Tuc main sequence and RGB was determined and is shown on the graph. The 47 Tuc data was shifted by $\Delta V=+1.88 \mathrm{mag}$ and $\Delta V-I=+0.105$ mag. These values were determined by requiring that the turnoff colors and the luminosities of the observed ZAHBs be coincident between the two clusters. 